\title{
Prevalence of proximal faecal stasis in active ulcerative colitis
}

\author{
M C Allison, $R$ Vallance
}

\begin{abstract}
Proximal faecal stasis may occur when faecal matter accumulates in the uninflamed colon above an area of active ulcerative colitis. This phenomenon is thought to be the cause of symptoms in some patients with distal disease. It is not known, however, how often patients with active distal colitis exhibit slow colonic transit. Fifty two consecutive patients with active ulcerative colitis each ingested 10 radioopaque markers nightly for 14 days. Minimum colonic transit times were derived from counts of retained markers shown by plain abdominal radiography on the 15 th day. The transit times for each patient were related to their disease extent and bowel frequency. Four patients had colonic transit times greater than one week and two others had evidence of relative stasis. The results indicate that approximately $10 \%$ of attacks of distal colitis are associated with faecal stasis.
\end{abstract}

Over one quarter of patients with active ulcerative protocolitis complain of hard stools in association with blood and mucus. These symptoms are frequently accompanied by tenesmus and a sensation of incomplete evacuation. This combination of symptoms has been attributed to stasis in the proximal colon and distal irritability. ${ }^{1}$ The common radiological finding of apparent accumulation of faeces in the right colon in patients with active ulcerative colitis would be consistent with this hypothesis. In a retrospective study, Jalan et al estimated that $16 \%$ of patients with active disease have proximal faecal stasis, as defined by the passage of hard stools and associated radiological evidence of faeces in the caecum and ascending colon. ${ }^{2} \mathrm{~A}$ radiographic finding of faecal accumulation in the right colon, however, does not necessarily mean that stasis is present. ${ }^{3}$ Rao et al have shown that proximal colonic transit is slowed in patients with active ulcerative colitis, and that transit through the distal colon is more rapid than normal. These phenomena balance one another, so that whole gut transit time in active ulcerative colitis is similar to that in patients with quiescent disease or healthy subjects. ${ }^{4}$

Lennard-Jones et al were the first to suggest

Departments of Medic and Radiology, Gartnave General Hospital

Glasgow

M C Alliso

Correspondence to Dr M C Allison, Departmen of Gastroenterology, Royal Infirmary, Glasgow G4 OSF.

Accepted for publication 4 April 1990 that proximal faecal stasis may be clinically important in some patients with active distal ulcerative colitis. ${ }^{5}$ They described six patients with coexistent constipation and rectal bleeding, two of whom developed subacute obstruction due to impacted faeces and barium in the ascending colon. They suggested that severe left sided colitis could act as a functional obstruction, leading to retention and inspissation of solid faeces in the uninflamed right colon. Subsequently, Cowan et al reported on a subgroup of patients with ulcerative colitis in whom faecal stasis in the proximal colon was thought to be responsible for slowing the transit of sulphasalazine and its metabolic products into the inflamed distal colon, thus delaying the therapeutic response to the drug. ${ }^{6}$ The conclusion from both of these studies was that seemingly paradoxical treatment with laxatives and a high fibre diet could benefit some patients with active distal ulcerative colitis.

This study was prompted by the lack of available guidelines for defining true faecal stasis in patients with active ulcerative colitis. We have used a dynamic marker technique to examine the prevalence of slow colonic transit in a consecutive group of these patients and have related the findings to their frequency of defecation and extent of disease.

\section{Methods}

\section{PATIENTS}

Sixty four consecutive patients with active ulcerative proctocolitis were considered for this study. The diagnosis had been established by sigmoidoscopy and rectal biopsy specimen in all cases. Active disease was defined as (i) altered bowel habit or presence of blood in stool, or both, and (ii) reddened and friable rectal mucosa identified by rigid sigmoidoscopy at the time of enrolment. Twelve patients were excluded for reasons given in the next paragraph. The remaining 52 patients all agreed to participate in the study. The anatomical extent of disease had been determined by endoscopy or barium enema within the previous two years in most patients; these investigations were performed after the study in patients in whom the disease extent was unclear at the time of enrolment. There were eight patients with proctitis alone, in whom the upper limit of disease was visible in the rectum at sigmoidoscopy on entry to the study. There were 22 patients with proctosigmoiditis (disease activity confined to the rectum and sigmoid colon); 12 patients with left sided disease (activity extending past sigmoid but not beyond mid-transverse colon); and 10 patients with more extensive disease. All patients received standard
Details of patient groups, bowel habits, and numbers of retained markers. The data are expressed as median (range)

\begin{tabular}{lllll}
\hline Disease extent & $\begin{array}{l}\text { No of patients } \\
\text { (sex distribution) }\end{array}$ & $\begin{array}{l}\text { Age } \\
\text { yrs) }\end{array}$ & $\begin{array}{l}\text { Mean No of } \\
\text { daily bowel } \\
\text { actions }\end{array}$ & $\begin{array}{l}\text { No of } \\
\text { retained } \\
\text { markers }\end{array}$ \\
\hline Proctitis alone & $8(7 \mathrm{~F} 1 \mathrm{M})$ & $33(21-54)$ & $1 \cdot 8(1-4 \cdot 1)$ & $33(15-111)$ \\
Proctosigmoiditis & $22(8 \mathrm{~F} 14 \mathrm{M})$ & $37(22-63)$ & $2 \cdot 8(0 \cdot 9-7 \cdot 8)$ & $15(1-105)$ \\
Left side of colon & $11(6 \mathrm{~F} 5 \mathrm{M})$ & $32(21-54)$ & $3 \cdot 2(1 \cdot 5-7 \cdot 9)$ & $15(0-70)$ \\
Extensive disease & $10(4 \mathrm{~F} 6 \mathrm{M})$ & $37(17-68)$ & $3 \cdot 4(2 \cdot 8-9 \cdot 1)$ & $14 \cdot 5(0-36)$ \\
\hline
\end{tabular}


treatment for active disease during the period of study. Four of the 52 patients were newly diagnosed. The remainder had had their disease for a median of six years (range $0 \cdot 5-12$ years). Six were receiving inpatient treatment; the others were recruited from the gastroenterology clinic. The age and sex distributions in these patient groups is shown in the Table.

\section{EXCLUSIONS}

Twelve patients were excluded because of factors that would have confounded or precluded transit studies. These included six patients who had required bowel preparation for colonoscopy or barium studies within the previous two months; four patients with severe illness, in whom urgent barium enema or surgery were imminent; and two patients taking non-specific antidiarrhoeal drugs.

\section{RADIO-OPAQUE MARKERS}

The markers were barium sulphate impregnated polyethylene pellets (Portex Ltd, Hythe, Kent) which are approximately cube shaped and have a mean weight of $27 \mathrm{mg}$; their use as markers for the transit of dietary residue through the human gut has been validated. ${ }^{7}$ The markers were treated with hot air from a hair drier to round off sharp corners, and were sieved through a coarse wire mesh to exclude uneven pellets.

\section{TRANSIT MEASUREMENTS}

The dynamic marker technique used in this study for measurement of transit has been described previously by one of us. ${ }^{3}$ Each patient ingested one capsule containing 10 markers each night for 14 nights and attended for a plain abdominal radiograph at $2 \mathrm{pm}$ on the 15 th day. The numbers of retained markers were determined from the radiograph. The minimum colonic transit time can be estimated by this method because an approximate equilibrium

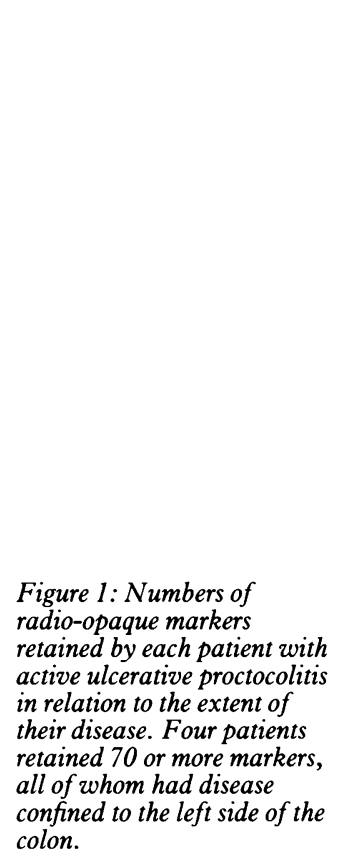

between markers ingested and excreted becomes established by the time the radiograph is taken. For example, if 50 markers are retained, the colonic transit time cannot be less than five days. An almost identical technique using ring shaped markers has been found by others ${ }^{8}$ to correlate well with the method of Cummings et al for the determination of whole gut transit time. ${ }^{7}$

Proximal faecal stasis was considered to be present in patients with 70 or more retained markers (equivalent to a minimum colonic transit time of one week). This definition was based on results obtained by an identical method in 22 previously studied healthy subjects. ${ }^{3}$ The numbers of retained markers in that group ranged from 2-74 (median value 24), and the counts in $90 \%$ of them fell within the range of 3-61.

Patients identified as having proximal faecal stasis were asked if they would consent to repeat transit studies after treatment of their active disease. The repeat studies were carried out at least three months after achievement of clinical and sigmoidoscopic remission.

\section{DIARY CARDS}

Each patient was asked to keep a diary card during the two week study period to encourage compliance with taking the marker capsules and to record details of bowel habit. The information requested for each study day was as follows: time of taking marker capsule; number of bowel actions; number of bowel actions resulting in formed stools; presence or absence of blood with stool.

\section{STATISTICAL ANALYSIS}

The numbers of retained markers in each group were not normally distributed. Thus the significance of observed differences between marker counts in each group was assessed by means of the Wilcoxon rank sum test for unpaired data. The relation between numbers of retained markers and bowel habit was examined using Spearman's rank correlation method.

\section{Results}

One patient, who did not complete the study due to the development of nausea after taking the marker capsules, was excluded from the analysis. Two other patients missed one capsule each during the early part of the study. They were not excluded because their marker counts were small, implying that all markers taken during the first week of the study would have been excreted by the time of the radiograph. Thus, results from 51 patients were available for analysis.

The median marker counts in each of the four groups are shown in the Table. There was a tendency for more markers to be retained by the patients with disease confined to the rectum compared with those with more extensive disease. Figure 1 illustrates the wide ranges of counts among the patients in each group. This observation would explain why no significant differences were found between any of the four groups. 
Figure 2: Plain abdominal radiograph from a patient with active proctosigmoiditis taken after she had ingested 10 markers daily for two weeks. There are 105 retained markers evenly distributed throughout the accumulated faeces.

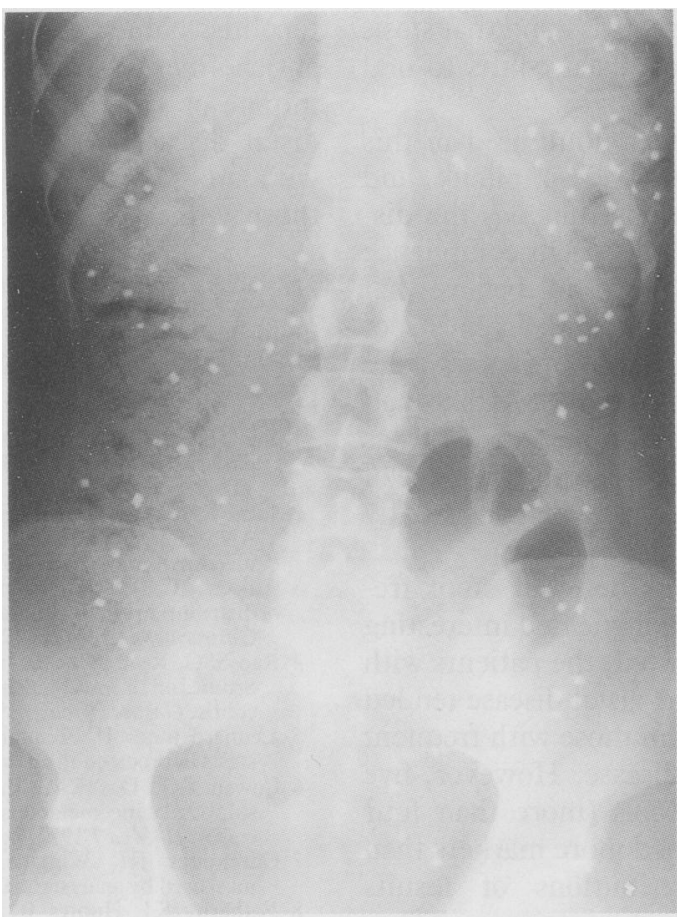

frequency was variable; and they were usually associated with blood and mucus. Similar symptoms were described by many of the patients without stasis.

A scatter diagram was constructed to examine the relation between speed of colonic transit and bowel frequency during the two week study period (Fig 3). No direct correlation between these two parameters was found $(r=-0 \cdot 11)$. Most patients recorded 20-60 bowel actions and retained 10-40 markers. Two other clusters of points were observed on this plot: a group of patients with very frequent stools exhibiting rapid transit (8-16 retained markers); and a group recording less than 40 bowel actions with evidence of relatively slow transit (50 or more markers retained). Thus, most points on the plot approximated to a parabolic distribution. The results in five patients, including three with faecal stasis, fell well outside this distribution; they had recorded more than four bowel actions daily, but had retained more markers than would be predicted from the scatter of results in the other patients.

Proximal faecal stasis was identified in four patients $(8 \%)$, none of whom had disease extending beyond the descending colon. Their retained markers were uniformly distributed in the uninflamed colon proximal to the diseased rectosigmoid region. Their minimum colonic transit times ranged from 7-11 days. The radiograph from one of these patients is shown in Figure 2. Three of these patients had recorded 57 bowel actions during the two week study period (mean $4 \cdot 1$ per day). The fourth recorded only $13(0.9$ per day). Three of the four patients found to have stasis in association with active disease underwent a repeat marker study after treatment. Their respective retained marker counts were 111,105 , and 89 during active disease; and 45, 53 , and 58 in remission.

The stool patterns described by the patients with stasis were not characteristic. Stools varied in consistency from loose, formed, or hard; their

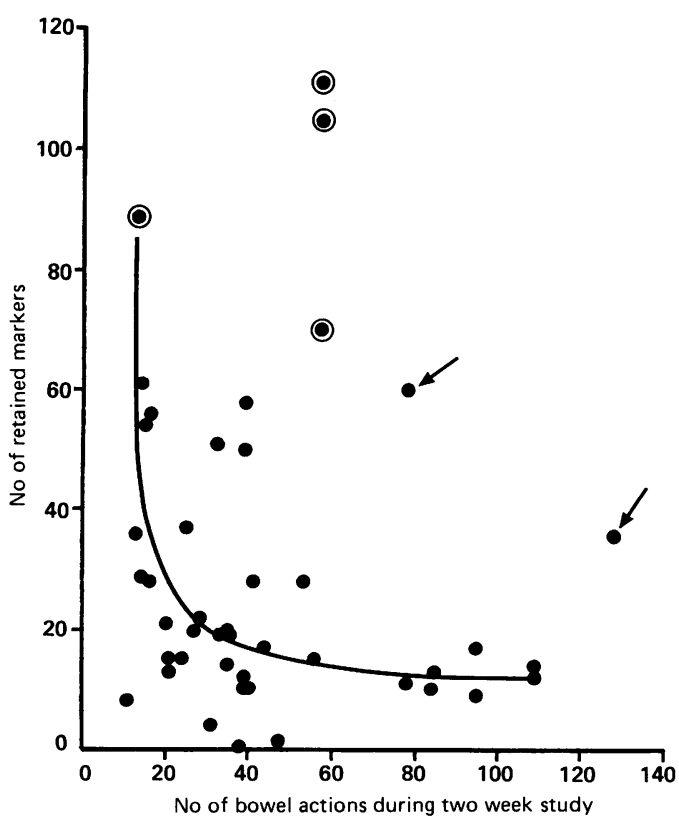

\section{Discussion}

The term 'proximal faecal stasis' has been used by several authors to describe accumulation of faecal residue above an area of active distal ulcerative colitis. ${ }^{1256}$ These reports differ in their definition of stasis. There is no doubt that proximal stasis prevailed in the six patients described in the initial report, ${ }^{5}$ because a mixture of faeces and barium persisted in the ascending colon several weeks after barium enema. Subsequent studies have defined faecal stasis as a reduction in bowel frequency associated with radiographic evidence of faeces in the proximal colon. ${ }^{26}$ The finding of proximal faecal accumulation on plain abdominal radiographs, however, does not necessarily mean that turnover of material in the right colon is slowed. ${ }^{3}$ Furthermore, the radiological interpretation of faecal loading in the right colon is rather subjective. Rao et al showed that transit through the right colon is generally slower in patients with active ulcerative colitis than in control subjects, ${ }^{4}$ and Black et al have shown prolonged whole gut transit in patients with disease confined to the rectum. ${ }^{9}$ Three questions remained unanswered - when does 'slow transit' become 'faecal stasis'; what is the prevalence of faecal stasis; and when does faecal stasis become clinically important?

The first two questions have been addressed in the present study. Faecal stasis has been defined on the basis of colonic transit measurements in healthy subjects, and similar measurements have been used to examine its prevalence in patients with active ulcerative colitis. The results suggest that about $10 \%$ of attacks of distal ulcerative colitis are associated with faecal stasis. The clinical importance of this observation remains uncertain, but such stasis could contribute to symptoms of constipation, tenesmus, and sensations of incomplete evacuation that are present in a proportion of patients with active disease. ${ }^{12910}$ Although faecal stasis is considered by some to be an uncommon sequela of distal colitis, ${ }^{5}$ it has been suggested by others that slow transit may 
predispose to ulcerative proctitis, ${ }^{9}$ and that stasis may delay the response of distal colitis to oral sulphasalazine. ${ }^{6}$

The dynamic marker technique used in this study has the advantages of simplicity and avoidance of stool collections, but has the disadvantage of dependence on patient compliance with marker ingestion. We do not believe that compliance difficulties led to misdiagnosis of faecal stasis because the retained markers were uniformly distributed in the faeces that had accumulated within the non-inflamed colon (as in Fig 2). Furthermore, repeat studies in three of the patients provided evidence of consistently slow transit.

Analysis of the relation between stool frequency and marker retention yielded interesting results. As would be expected, the patients with lowest stool frequency and distal disease tended to retain more markers than those with frequent diarrhoea and extensive disease. However, five patients with frequent stools (more than four bowel actions daily) retained more markers than expected from the distributions of results observed in the rest of the patients. These findings would be consistent with the interpretation that a motility disorder exists in a subgroup of patients with active colitis, leading to an inappropriate degree of proximal faecal retention despite the passage of frequent stools associated with blood and mucus. Several motility disturbances have been described in active ulcerative colitis, including decreased intraluminal pressure in the descending colon, ${ }^{11}$ increased propagating contractions, ${ }^{11}$ increased rectal sensitivity and hyper-reactivity to distension,,$^{12}$ and poor rectal compliance. ${ }^{1314}$

The importance of recognising and treating proximal faecal stasis remains unknown, but the prescribing of certain drugs could be influenced by awareness that this phenomenon occurs in some patients with active distal ulcerative proc- tocolitis. Non-specific antidiarrhoeal treatment, anticholinergic drugs, or opiate analgesics could initiate or worsen faecal stasis in patients with distal disease ${ }^{4}$ in addition to being associated with an increased risk of toxic megacolon in those with extensive disease. ${ }^{15}$

The authors acknowledge the assistance of their research nurse, Ms V Woolfson, who was supported by the Greater Glasgow Health Board. We are grateful to Dr J G Allan, Dr K E L McColl, and Dr P Mills for allowing us to study their patients.

1 Rao SSC, Holdsworth CD, Read NW. Symptoms and stoo pao SSC, Holdsworth CD, Rearive colitis. Gut 1988; 29 . patterns

2 Jalan KN, Walker RJ, Prescott RJ, Butterworth STG, Smith AN, Sircus W. Faecal stasis and diverticular disease in ulcerative colitis. Gut 1970; 11: 688-96.

3 Allison MC, Dick R, Pounder RE. A controlled study of faeca distribution in ulcerative colitis and proctitis. Scand $\mathcal{F}$ Gastroenterol 1987; 22: 1277-80.

4 Rao SSC, Read NW, Brown C, Bruce C, Holdsworth CD. Studies on the mechanism of bowel disturbance in ulcerative colitis. Gastroenterology 1987; 93: 934-40.

5 Lennard-Jones JE, Langman MJS, Avery Jones F. Faecal tasis in proctocolitis. Gut 1962; 3: 301-6.

6 Cowan GO, Das KM, Eastwood MA. Further studies of sulphasalazine metabolism in the treatment of ulcerative colitis. Br Med F 1977; ii: 1055-7.

7 Cummings JH, Wiggins HS. Transit through the gut measured by analysis of a single stool. Gut 1976; 17: 219-23.

8 Fotherby KJ, Hunter JO. Idiopathic slow-transit constipation: whole gut transit times, measured by a new simplified method, are not shortened by opioid antagonists. Alimen Pharmacol Therap 1987; 1: 331-8.

9 Black DA, Ainley CC, Senapati A, Thompson RPH. Transit time in ulcerative proctitis. Scand $\mathcal{F}$ Gastroenterol 1987; 22: 872-6.

10 Engel GL. Studies of ulcerative colitis. II. The nature of the somatic process and the adequacy of psychosomatic hyposomatic process and the adequacy

11 Chan SS, Reddy SN, Villaneuva J, et al. Colonic motility and transit of intraluminal contents in ulcerative colitis. Gastrotransit of intraluminal cont

12 Farthing MJG, Lennard-Jones JE. Sensibility of the rectum io distension and the anorectal distension reflex in ulcerative colitis. Gut 1978; 19: 64-9.

13 Rao SSC, Read NW, Davison PA, Bannister JJ, Holdsworth $\mathrm{CD}$. Anorectal sensitivity and responses to rectal distension in patients with ulcerative colitis. Gastroenterology 1987; 93: $1270-5$

14 Denis PH, Colin R, Galmiche JP, et al. Elastic properties of the rectal wall in normal adults and in patients with ulcerative rectal wall in normal adults and in pa

15 Huizenga KA, Schroeder KW. Gastrointestinal complication of ulcerative colitis and Crohn's disease. In: Kirsner JB Shorter RG, eds. Inflammatory bowel disease. Philadelphia: Lea and Febiger, 1988: 257-80. 